\title{
Impact of false-positive newborn metabolic screening results on early health care utilization
}

\author{
Ellen A. Lipstein, $M D^{1}$, James M. Perrin, MD ${ }^{1,2}$, Susan E. Waisbren, PhD ${ }^{2,3}$, \\ and Lisa A. Prosser, MS, PhD $D^{4,5}$
}

\begin{abstract}
Purpose: To analyze the association between false-positive newborn screening results and health care utilization. Methods: We surveyed parents regarding their children's health care utilization. Parents of children who received false-positive newborn screening results were primarily enrolled by a screening laboratory in Pennsylvania. Parents of children with normal results were recruited through the Massachusetts birth registry. We used bivariate tests and multivariate regression to assess the association between newborn screening results and primary care utilization, emergency room use, and hospitalization by the age of 6 months. Results: Our sample included 200 children with falsepositive results and 137 with normal results. Variation in recruitment strategies led to sample children with false-positive results being more likely to be non-white, have unmarried parents, and be of lower socioeconomic status. After adjusting for significant covariates, such as age, race, and socioeconomic status, there were no significant associations between newborn screening results and child health care utilization. Conclusions: Despite the reported negative psychosocial effects of false-positive results, our study found no impact on early health care utilization. These results may assist in economic analyses of newborn screening as they suggest that medical costs associated with falsepositive results are limited to the cost of diagnostic testing and follow-up. Genet Med 2009:11(10):716-721.
\end{abstract}

Key Words: health services utilization, neonatal screening, falsepositive results, metabolic diseases, genetic diseases

$\mathrm{D}$ uring the past decade, newborn screening programs for metabolic disorders have grown rapidly in large part because of increasing use of tandem mass spectrometry (MS/MS). Between 1995 and 2005, the average state added 19 disorders to its newborn screening program. ${ }^{1}$ Despite the high sensitivity and specificity of screening with MS/MS, ${ }^{2}$ expanded screening, such as that recommended by the American College of Medical Genetics Newborn Screening Expert Group, ${ }^{3}$ will lead to an increased number of infants with false-positive results because of the low positive predictive values inherent when screening for multiple independent diseases of low prevalence. Estimates

From the ${ }^{1}$ Center for Child and Adolescent Health Policy, MassGeneral Hospital for Children; ${ }^{2}$ Harvard Medical School; ${ }^{3}$ Metabolism Program, Children's Hospital Boston, Boston, Massachusetts; ${ }^{4}$ Division of General Pediatrics, Child Health Evaluation and Research Unit, University of Michigan Health System, Ann Arbor, Michigan; and ${ }^{5}$ Department of Ambulatory Care and Prevention, Center for Child Health Care Studies, Harvard Medical School and Harvard Pilgrim Health Care, Boston, Massachusetts.

Ellen A. Lipstein, MD, Center for Child and Adolescent Health Policy, MassGeneral Hospital for Children, 50 Staniford Street, Suite 901, Boston, MA 02114. E-mail: elipstein@partners.org.

Disclosure: The authors declare no conflict of interest

Submitted for publication March 9, 2009.

Accepted for publication June 19, 2009.

Published online ahead of print August 5, 2009

DOI: $10.1097 /$ GIM.0b013e3181b3a61e for the annual number of false-positive results in the United States range from 2500 to $>51,000 .{ }^{1}$ Economic analyses of newborn screening find expanded screening with MS/MS to be cost effective, in part because, once a program owns a tandem MS/MS, screening for additional diseases using mass spectrometer adds very little incremental cost. ${ }^{4-7}$ However, these studies generally assume the cost of a false-positive result to be only the cost of confirmatory testing and care during the confirmatory period.

The true impact of false-positive newborn screening tests is just beginning to be well described. Early screening programs for phenylketonuria showed poor parental understanding of false-positive results and a tendency for parents of such children to perceive their children as medically vulnerable. ${ }^{8}$ More recent research has demonstrated associations between false-positive results and increased parental stress, mothers' perceptions that their children with false-positive results require increased parental care, and a trend toward increased hospitalization. ${ }^{9,10}$ Long-lasting psychological stress has been seen in families of children with false-positive screening tests. ${ }^{11,12}$ Studies such as these have led to speculation that false-positive results may lead to increased perceptions of child vulnerability, ${ }^{1,13,14}$ which have been associated with increased health care utilization in other childhood conditions. ${ }^{15-18}$ Indeed, early work suggested that increased hospitalization is seen among children with falsepositive newborn screen results ${ }^{10}$; however, the associations between false-positive newborn screening results and health care utilization have not been well described. In this study, we hypothesized that a false-positive result would be associated with increased outpatient and inpatient health care utilization in early childhood.

\section{MATERIALS AND METHODS}

\section{Sample}

Parents of children with false-positive newborn screen results, defined as the initial result being abnormal or inconclusive for any of 20 biochemical disorders, were invited to participate after a referral was made for additional confirmatory testing. The authors planned to recruit all subjects from within New England but were unable to access information for New England children with false-positive results who did not present for evaluation at a metabolic center. Therefore, we contracted with a private laboratory that conducts newborn screening for more than $99 \%$ of birthing hospitals in Pennsylvania, a state demographically similar to Massachusetts, ${ }^{19}$ to recruit and interview parents of children with false-positive newborn screen results. Participants were enrolled by this laboratory and by clinical metabolic centers in New England, between 1999 and 2005. In Pennsylvania, recruitment materials were sent to the families of all known cases of false-positive newborn screen results. In New England, recruitment materials were sent to families who sought follow-up testing at one of the clinical metabolic centers. Parents were sent a letter explaining the study $\geq 6$ months after the diagnosis of a metabolic disorder had 
been ruled out. Those who did not opt-out by returning a response card were called to participate in a telephone interview.

The children with normal test results, confirmed by parent self-report, were 6- to 12-month-old children selected randomly from the Commonwealth of Massachusetts Department of Public Health Birth Registry of Vital Records and Statistics for live births between February 1999 and September 2005. These birth registry data include only children born to married couples. ${ }^{10}$ Families were recruited by letter followed by a telephone call if an opt-out card was not returned. All participants were sent a small baby gift, worth $\$ 7.00$, after completing the interview.

Exclusion criteria for both groups were children who had died and newborns whose birth weight was $<2500 \mathrm{~g}$. The latter criterion avoided recruitment of significantly premature infants who frequently experience transient newborn screening abnormalities. Although both parents were invited to participate in the survey, for this study, we used only data collected from mothers because when there were data from only one parent it was the mother in almost all cases. The institutional review boards of Children's Hospital Boston and The Children's Hospital of Philadelphia approved the study.

\section{Data collection}

We conducted a one-time 30-minute telephone survey. Complete details of data collection have been reported previously. $9,10,20$ We asked mothers "in the past year, how many times did you visit a primary care physician for your child's care" and analyzed these data as a count of the number of visits. Mothers were also asked "whether [they] had ever taken [their] child to the emergency room" and the dates of such visits. These data were dichotomized as children who had or had not been to the emergency room. Mothers provided the dates of all overnight hospitalizations, which allowed us to classify children according to whether they were hospitalized in the first 6 months of life. Finally, we asked for the number of times the child had visited a specialist, exclusive of any visits associated with confirming the false-positive screening result.

We collected data on sociodemographic characteristics including parental education and occupation (which makeup the Hollingshead Index of Social Position ${ }^{21}$ ), and marital status, which we dichotomized as married or unmarried. Parents were also asked whether their "child has any health problems" and to name the type of problem. In addition, participants completed the Parental Stress Index, a 36-item questionnaire from which we used the total stress score. ${ }^{9,10,22}$

\section{Data analysis}

We initially conducted bivariate analyses to compare health care utilization in children with false-positive newborn screen results versus normal screen results. We used nonparametric tests (Kolmogorov-Smirnov test ${ }^{23}$ ) to assess the associations between newborn screen result and both primary care and specialist use. We used $\chi^{2}$ tests to assess the proportions who were hospitalized in the first 6 months and who had ever been to the emergency room. We used SAS 9.1 (SAS Institute Inc., Cary, NC) for all statistical analyses.

We then developed regression models for each dependent variable, using confounding covariates, defined as those variables (such as child age, sex, and race, Hollingshead Social Position or maternal marital status) that showed a bivariate relationship with that dependent variable and with screening result at $P \leq 0.2$. We did not include maternal education in any model because the Hollingshead Social Position incorporates maternal education. In addition, because we could not verify the reported health problems, this variable was used only in sensitivity analyses, so as not to control for parent perceptions of vulnerability that may influence reporting of both child health problems and child health care utilization. Poisson regression was used for the association between newborn screen results and primary care utilization. We used multivariate logistic regression models to assess the relationship between newborn screen results and emergency room use or hospital admission.

Children who were missing a variable used in an analysis were excluded from that particular analysis. The most frequently missing variable was Hollingshead Index of Social Position, missing for four $(2 \%)$ children with false-positive results and zero children with normal newborn screening results.

We performed three different sensitivity analyses. In the first, we assumed that all children who were not yet 6 months at the time of interview were hospitalized before their 6-month birthday. In the second, we eliminated all unmarried mothers from the false-positive cohort and reassessed all associations between the dependent variables and newborn screening results. We conducted these first two sensitivity analyses to help determine the influence of the variation in our recruitment strategy between the children with normal and the children with falsepositive results. Finally, we reanalyzed all multivariate regressions with an additional covariate, indicating whether the child's mother reported the child had any health problems to account for any influence true disease may have had on health care utilization.

\section{RESULTS}

\section{Participants}

The final sample included 200 children with a false-positive newborn screen and 137 with a normal newborn screen. The response rates were $75 \%$ among parents of children with falsepositive results and $63 \%$ among parents of children with normal results. A total of $87.9 \%$ of participants with false-positive results were from Pennsylvania (Table 1).

The children in the false-positive group had a mean age of 12.9 months compared with 6.4 months for the normal screen group. Children in our sample with false-positive screens were more likely than children with normal screens to be non-white, have unmarried parents, come from families of lower socioeconomic status, and have reported health problems. In addition, mothers whose child had a false-positive result scored higher on the Parental Stress Index (Table 1).

\section{Health care utilization}

Children with false-positive screens had a median of 8 (interquartile range: $6-12$ ) primary care visits compared with a median of 6 (interquartile range, 5-8) for their normal screen counterparts $(P<0.0001)$. After adjusting for children's age, which was the only significant covariate for primary care utilization, the adjusted rate ratio was 1.10 (95\% CI: 0.98-1.22; $P=0.09$ ) (Table 2).

Four $(2.0 \%)$ children with false-positive screens had been to a specialist, each with no more than one visit. In contrast, 14 $(10.5 \%)$ children with normal newborn screens had been to a specialist with the number of visits varying from 1 to 15 (mean 3.5; mode 1). These differences in specialist utilization were not significant $(P=0.63)$.

In unadjusted analyses, children with false-positive screens were more likely to have ever visited the emergency room (28.6\% vs. $11.7 \% ; P<0.001)$. After adjusting for the children's age, the odds ratio (OR) for children with a false-positive result 
Table 1 Characteristics according to newborn screening result (unadjusted)

\begin{tabular}{|c|c|c|c|}
\hline & $\begin{array}{c}\text { False } \\
\text { positive } \\
(n=200)\end{array}$ & $\begin{array}{c}\text { Normal } \\
\text { screen } \\
(n=137)\end{array}$ & $P$ \\
\hline \multicolumn{4}{|l|}{ Sociodemographic characteristics } \\
\hline Age at time of interview (mo) & 12.9 & 6.4 & $<0.0001$ \\
\hline Female $(\%)$ & 47.5 & 46.7 & 0.88 \\
\hline Race/ethnicity (\%) & & & 0.0001 \\
\hline Non-Hispanic white & 79.0 & 92.7 & \\
\hline Non-Hispanic black & 12.5 & 0.7 & \\
\hline Hispanic & 5.5 & 1.5 & \\
\hline Others & 3.0 & 5.2 & \\
\hline Health problems $(\%)$ & & & 0.01 \\
\hline Overall & 33.5 & 20.7 & \\
\hline Prematurity & 6.5 & 1.5 & \\
\hline Jaundice & 9.0 & 5.9 & \\
\hline Respiratory problems & 4.5 & 1.5 & \\
\hline Ear problems & 4.5 & 0.7 & \\
\hline Gastrointestinal problems & 3.0 & 4.4 & \\
\hline Others & 6.0 & 6.7 & \\
\hline Place of residence $(\%)$ & & & $<0.0001$ \\
\hline New England & 12.1 & 100 & \\
\hline Pennsylvania & 87.9 & 0 & \\
\hline $\begin{array}{c}\text { Hollingshead social } \\
\operatorname{class}^{a}(\%)\end{array}$ & & & $<0.0001$ \\
\hline 1,2 , or 3 & 63.3 & 91.2 & \\
\hline 4 or 5 & 27.7 & 8.8 & \\
\hline Parents married (\%) & 79.0 & 100 & $<0.0001$ \\
\hline Maternal education (\%) & & & $<0.0001$ \\
\hline High school or less & 37.5 & 9.5 & \\
\hline Greater than high school & 62.5 & 90.5 & \\
\hline $\begin{array}{l}\text { Mother's Parental Stress } \\
\text { Index Score }\end{array}$ & 70.3 & 59.2 & $<0.0001$ \\
\hline \multicolumn{4}{|l|}{ Health care utilization } \\
\hline $\begin{array}{l}\text { Number of outpatient visits, } \\
\text { median }\end{array}$ & 8 & 6 & $<0.0001$ \\
\hline Ever visited a specialist (\%) & 2 & 10.5 & 0.63 \\
\hline $\begin{array}{l}\text { Ever visited an emergency } \\
\text { room (\%) }\end{array}$ & 28.6 & 11.7 & $<0.001$ \\
\hline $\begin{array}{l}\text { Hospitalized prior to } 6 \\
\text { months of age }(\%)\end{array}$ & 15.4 & 8.8 & 0.07 \\
\hline
\end{tabular}

$\overline{{ }^{a} \text { Incorporates level of education and occupational status. Lower numbers indicate }}$ higher socioeconomic status.

${ }^{b}$ Higher number indicates greater stress. having ever received care in an emergency room compared with children with a normal screening result was 1.31 (95\% CI: $0.57-3.03 ; P=0.53$ ). Further adjustment for significant sociodemographic variables did not significantly change the results $(P=0.88)$, although parent marital status trended toward statistical significance (Table 2).

A total of $15.4 \%$ of the children with false-positive newborn screens had been hospitalized by 6 months of age compared with $8.8 \%$ of the children with normal screens. The OR for children with a false-positive screen being hospitalized in the first 6 months, compared with children with normal screens, was not significant in the unadjusted analysis (OR 1.89; $95 \%$ CI: $0.93-3.85 ; P=0.08$ ). After adjusting for significant sociodemographic differences, specifically children's sex, race/ ethnicity, and family social position, the effect of newborn screening result remained nonsignificant (OR 1.51; 95\% CI: $0.70-3.22 ; P=0.29$ ). Child sex was significantly related to odds of hospitalization (Table 2).

\section{Sensitivity analyses}

Among the children who had not been hospitalized, there were 13 normal screen children and 1 false-positive child who were just younger than 6 months at the time of interview. Conservatively, assuming that all 14 of these children were hospitalized before 6 months of age, our results remained nonsignificant.

Because the children with normal screens all had married parents, we assessed whether this difference between the groups may have masked any association between newborn screen results and health care utilization. After excluding all children with unmarried mothers (from the false-positive group), the results for all utilization outcomes (primary care, specialists, emergency room, and hospitalization before 6 months) remained nonsignificant. Finally, the addition of a covariate indicating that the child had a history of health problems did not change the significance of any results.

\section{DISCUSSION}

As newborn screening programs expand to include more disorders, many children will experience false-positive test results. ${ }^{1}$ In our study, such results were not associated with increased health care utilization early in life. Although falsepositive tests have been associated with higher parental stress, ${ }^{9}$ this increased stress does not appear to lead to more health care utilization.

This information has particular relevance for cost-effectiveness evaluations of newborn screening. The majority of such evaluations of expanded newborn screening have limited the costs of a false-positive test to the costs that occur before diagnostic confirmation. ${ }^{4-7}$ These studies assume that after a positive test result has been proven to be a false positive no further costs are associated with that outcome. Our study suggests that, particularly for outpatient costs, this assumption is appropriate.

Early studies of population-based pediatric screening programs showed increased health care utilization after false-positive results ${ }^{8}$ and parental misunderstanding of results leading to unnecessary restriction of children's activities. ${ }^{24}$ The differences between these studies and the results reported here could suggest that, as newborn screening has expanded, parental understanding of such screening and parental interpretation of its results have improved. One could hypothesize that today's physicians and nurses more effectively communicate test results to families than did their predecessors. Studies of communica- 
Table 2 Associations between false-positive newborn screening result and early health care utilization adjusted for significant sociodemographic confounders

$95 \%$ confidence interval for

rate ratio

Adjusted RR for outpatient utilization in the previous year

False-positive newborn screen result

One month increase in child's age

$0.98-1.22$

Adjusted OR for ever using the emergency room

False-positive newborn screen result

One month increase in child's age

Hollingshead Social Position $<4^{a}$

Parents married

Adjusted ORs for hospitalization prior to 6 months of age

False-positive newborn screen result

Hollingshead Social Position $<4^{a}$

Sex: female

Race: white
$1.04-1.06$

$0.50-2.81$

0.88

$1.04-1.22$

$<0.01$

$0.55-2.07$

0.85

$0.20-1.01$

0.56
$0.70-3.22$

$0.28-1.22$

$1.13-4.42$

$0.24-1.29$

${ }^{a}$ Incorporates level of parent education and occupational status. Lower numbers indicate higher socioeconomic status.

$\mathrm{RR}$, rate ratios; $\mathrm{OR}$, odds ratios.

tion around newborn screening results, however, do not support this hypothesis. Researchers have consistently shown providers' ability to communicate about newborn screening is poor in both training and primary care settings. ${ }^{25-29}$ It may be that physicians do not communicate the potentially serious nature of the initial positive screening test to families, thus parents have no reason for concern. This idea is supported by the fact that, as detailed elsewhere, in our study sample many parents of children with false-positive results did not know the correct reason for their child's follow-up testing. ${ }^{9}$

Alternatively, it may be that the association between falsepositive newborn screening results and health care utilization varies by disorder, with positive tests for more life-threatening disorders leading to greater utilization. In such a situation, if our sample was biased toward less serious disorders, we may have underestimated the impact of false-positive results on early health care utilization. Similarly, if our sample of children with normal results had particularly high rates of health care utilization, these levels could mask any increased utilization among our false-positive sample. However, both groups had lower than average rates of specialist use, ${ }^{30}$ making it unlikely that the normal screen group had unusually high utilization rates.

Earlier studies, using a subset of our study sample, demonstrated increased parental stress among parents of children with false-positive newborn screening results. ${ }^{9,10}$ Other authors have also found an association between false-positive newborn screen results and negative psychosocial effects. ${ }^{31,32}$ We hypothesized that such psychosocial effects could lead to parents perceiving children with false-positive test results as vulnerable, which in turn may lead to increased health care utilization. ${ }^{16,17}$ Our results contradict this idea and show that the increased parental stress associated with false-positive results in other studies $^{9}$ may not be associated with perceptions of child vulnerability that would be expected to lead to increased health care utilization. These results are consistent with a recent study, using a portion of our sample, which, using economic valuation methods, demonstrated that parents who have experienced a false-positive result have high tolerance for such results. ${ }^{20}$

This study was limited by its short time horizon. Parents were interviewed when their children were quite young, thus any long-term differences in health care utilization would not have been seen. However, previous work has shown that perceptions of vulnerability decrease over time. ${ }^{33}$ This suggests that differences in utilization due to vulnerability should have been captured in the time frame we used. Furthermore, as in any survey, parents may have been biased in their recollection of health care utilization, health problems or, in the case of the families assumed to have normal screens, newborn screening results. However, if the false-positive screens resulted in persistent parental concerns, we would expect any differential response bias to favor both increased utilization among children with false-positive screens and an increased likelihood of parents correctly recalling the child's test results. Similarly, we chose not to control for the reported presence of health problems because of concern that such reports may be related to parental concerns, potentially triggered by newborn screening experiences rather than children's actual health. We acknowledge that some, if not all, of the reported health problems may reflect actual disease and these children would be expected to use more health care. However, sensitivity analyses adjusting for such health problems found no associations between newborn screening result and health care utilization.

In addition, children with false-positive results were significantly older than children with normal screens. We recognize that older children have greater opportunity for health care utilization but also that younger children have more frequent scheduled primary care use. For this reason, we attempted to compensate for differences in age through the inclusion of the child's age as a covariate in all regression analyses except hospitalization in the first 6 months of life. In addition to 
differences in mean age, there were significant sociodemographic differences between our two samples. We believe these differences reflect our varied recruitment strategies rather than true population differences between children who have normal and false-positive newborn screening results. Adjusting for these sociodemographic differences through regression analyses did not change our results but may not fully address any differential access to care that may influence utilization. Moreover, in sensitivity analyses our results were robust to the exclusion of unmarried mothers from the false-positive group. Finally, we did not collect information regarding the specific false-positive result received. Therefore, we can not assess whether a specific subset of false-positive results may be associated with increased health care utilization.

Adjusting for possible confounders reduced the association between a false-positive newborn screening result and health care utilization; this suggests that there may be sociodemographic disparities in health care utilization after a false-positive newborn screening. Further research in this area should specifically address whether such disparities exist and possible mechanisms, such as that some families may not receive adequate counseling and reassurance after a false-positive newborn screening result and hence may be more likely to seek additional medical care.

This is the largest study to date of children with false-positive newborn screening results. The sample afforded us with adequate power to detect a rate ratio as small as 1.17 , one to two extra visits annually, for outpatient care. Conversely, we were underpowered to detect differences in emergency room and inpatient utilization, events that are much less common than primary care visits. A relatively small study such as this one provides point estimates that can be used to determine sample sizes for future studies. For a study to have $80 \%$ power to detect a $50 \%$ difference in the odds of hospitalization in the first 6 months, or an OR of 1.5, would require more than 2200 subjects. Similarly, more than 3400 would be required to detect a $50 \%$ difference in the odds of emergency room use. To detect a smaller difference in utilization, however, one that may be significant on a population basis would require an even larger sample size. Additional research in this area should include follow-up studies with larger sample sizes and longer time frames.

\section{CONCLUSION}

The advancement of technologies such as tandem MS/MS and DNA microarrays is likely to lead to continued expansion of newborn screening. ${ }^{34}$ This expansion should direct more attention to the impact of false-positive results. Our results suggest that false-positive results have little impact on early health care utilization and may therefore have little economic impact. However, it is unclear if this reflects parental understanding or misunderstanding of false-positive results. As education materials are developed for future screening programs, a better understanding of why the false-positive result has little impact on health care utilization may help physicians counsel families with such results.

\section{ACKNOWLEDGMENTS}

This study was funded by Grants from Maternal and Child Health Bureau (5H46 MC00158) and the Ethical, Legal, and Social Implications (ELSI) division of the Human Genome Project, National Institutes of Health (RO1 HG02085). Dr Lipstein's effort was supported by the Health Resources and Ser- vices Administration, Grant T32 HP10018 to the Harvard Pediatric Health Services Research Fellowship Program.

Presented in part at the Pediatric Academic Societies Meeting, Baltimore, Maryland on May 5, 2009. The authors acknowledge Dionne Graham, PhD, for her statistical assistance and the many parents who participated in this study.

\section{REFERENCES}

1. Tarini BA, Christakis DA, Welch HG. State newborn screening in the tandem mass spectrometry era: more tests, more false-positive results. $\mathrm{Pe}$ diatrics 2006;118:448-456.

2. Schulze A, Lindner M, Kohlmuller D, Olgemoller K, Mayatepek E, Hoffmann GF. Expanded newborn screening for inborn errors of metabolism by electrospray ionization-tandem mass spectrometry: results, outcome, and implications. Pediatrics 2003;111(6 Pt 1):1399-1406.

3. Watson MS, Mann MY, Lloyd-Puryear MA, Rinaldo P, Howell RR. Newborn screening: toward a uniform screening panel and system-executive summary. Pediatrics 2006;117:S296-S307.

4. Insinga RP, Laessig RH, Hoffman GL. Newborn screening with tandem mass spectrometry: examining its cost-effectiveness in the Wisconsin Newborn Screening Panel. J Pediatr 2002;141:524-531.

5. Carroll AE, Downs SM. Comprehensive cost-utility analysis of newborn screening strategies. Pediatrics 2006;117(5 Pt 2):S287-S295.

6. Venditti LN, Venditti CP, Berry GT, et al. Newborn screening by tandem mass spectrometry for medium-chain Acyl-CoA dehydrogenase deficiency: a cost-effectiveness analysis. Pediatrics 2003;112:1005-1015.

7. Schoen EJ, Baker JC, Colby CJ, To TT. Cost-benefit analysis of universal tandem mass spectrometry for newborn screening. Pediatrics 2002;110: 781-786.

8. Rothenberg MB, Sills EM. Iatrogenesis: the PKU anxiety syndrome. $J \mathrm{Am}$ Acad Child Psychiatry 1968;7:689-692.

9. Gurian EA, Kinnamon DD, Henry JJ, Waisbren SE. Expanded newborn screening for biochemical disorders: the effect of a false-positive result. Pediatrics 2006;117:1915-1921.

10. Waisbren SE, Albers S, Amato S, et al. Effect of expanded newborn screening for biochemical genetic disorders on child outcomes and parental stress. JAMA 2003;290:2564-2572.

11. Fyro K. Neonatal screening: life-stress scores in families given a falsepositive result. Acta Paediatr Scand 1988;77:232-238

12. Fyro K, Bodegard G. Four-year follow-up of psychological reactions to false positive screening tests for congenital hypothyroidism. Acta Paediatr Scand 1987;76:107-114.

13. Hewlett J, Waisbren SE. A review of the psychosocial effects of falsepositive results on parents and current communication practices in newborn screening. J Inherit Metab Dis 2006;29:677-682.

14. Farrell MH, Farrell PM. Newborn screening for cystic fibrosis: ensuring more good than harm. $J$ Pediatr 2003;143:707-712.

15. Allen EC, Manuel JC, Legault C, Naughton MJ, Pivor C, O'Shea TM. Perception of child vulnerability among mothers of former premature infants. Pediatrics 2004;113:267-273.

16. Levy JC. Vulnerable children: parents' perspectives and the use of medical care. Pediatrics 1980;65:956-963.

17. Janicke DM, Finney JW. Children's primary health care services: socialcognitive factors related to utilization. J Pediatr Psychol 2003;28:547-557.

18. Janicke DM, Finney JW, Riley AW. Children's health care use: a prospective investigation of factors related to care-seeking. Med Care 2001;39:9901001 .

19. U.S. Census Bureau. United States-Fact Sheet-American FactFinder. Available at: http://factfinder.census.gov. Accessed January 15, 2009.

20. Prosser LA, Ladapo JA, Rusinak D, Waisbren SE. Parental tolerance of false-positive newborn screening results. Arch Pediatr Adolesc Med 2008; 162:870-876.

21. Miller DC, Salkind NJ. Handbook of research design and social measurement, 5th ed. Newbury Park, CA: Sage Publications, 1991:351-359.

22. Abidin RR. Parenting Stress Index (PSI), 3rd ed. Odessa, FL: Psychological Assessment Resources, Inc.; 1995.

23. Sheskin DJ. Handbook of parametric and nonparametric statistical procedures. New York: Chapman and Hall; 2000.

24. Hampton ML, Anderson J, Lavizzo BS, Bergmen AB. Sickle cell "nondisease." A potentially serious public health problem. Am J Dis Child 1974; 128:58-61.

25. Davis TC, Humiston SG, Arnold CL, et al. Recommendations for effective newborn screening communication: results of focus groups with parents, providers, and experts. Pediatrics 2006;117(5 Pt 2):S326-S340.

26. Farrell MH, La Pean A, Ladouceur L. Content of communication by pediatric residents after newborn genetic screening. Pediatrics 2005;116:14921498.

27. Gennaccaro M, Waisbren SE, Marsden D. The knowledge gap in expanded 
newborn screening: survey results from paediatricians in Massachusetts. $J$ Inherit Metab Dis 2005;28:819-824.

28. La Pean A, Farrell MH. Initially misleading communication of carrier results after newborn genetic screening. Pediatrics 2005;116:1499-1505.

29. Farrell M, Deuster L, Donovan J, Christopher S. Pediatric residents' use of jargon during counseling about newborn genetic screening results. Pediatrics 2008; $122: 243-249$.

30. Kuhlthau K, Nyman RM, Ferris TG, Beal AC, Perrin JM. Correlates of use of specialty care. Pediatrics 2004;113(3 Pt 1):e249-e255.

31. Tluczek A, Koscik RL, Farrell PM, Rock MJ. Psychosocial risk associated with newborn screening for cystic fibrosis: parents' experience while awaiting the sweat-test appointment. Pediatrics 2005;115:1692-1703.

32. Baroni MA, Anderson YE, Mischler E. Cystic fibrosis newborn screening: impact of early screening results on parenting stress. Pediatr Nurs 1997;23: 143-151.

33. McCormick MC, Brooks-Gunn J, Workman-Daniels K, Peckham GJ. Maternal rating of child health at school age: does the vulnerable child syndrome persist? Pediatrics 1993;92:380-388.

34. Green NS, Pass KA. Neonatal screening by DNA microarray: spots and chips. Nat Rev Genet 2005;6:147-151. 